\title{
FERTIRRIEGO EN EL RENDIMIENTO DE HÍBRIDOS DE TOMATE PRODUCIDOS EN INVERNADERO ${ }^{1}$
}

\author{
Gustavo Quesada-Roldán², Floria Bertsch-Hernández ${ }^{3}$
}

\begin{abstract}
RESUMEN
Fertirriego en el rendimiento de híbridos de tomate producidos en invernadero. El objetivo del presente trabajo fue evaluar el efecto de diferentes programas de fertirriego, sobre la producción de híbridos de tomate en invernadero. De octubre 2008 a marzo 2009, se sembraron dos híbridos de tomate (FB-17 y DRD-8108) en invernadero en la Estación Experimental Agrícola Fabio Baudrit Moreno en Alajuela, Costa Rica, para evaluar su respuesta a estrategias de fertirriego, a partir de recomendaciones de dosis recopiladas de la literatura (LIT), curvas de absorción (CA) y producto del ajuste de la información aportada por los tratamientos previos (AJU). No se encontraron diferencias estadísticas significativas en el rendimiento comercial en ninguno de los tres tratamientos de fertilización; únicamente se presentaron diferencias entre los híbridos. El híbrido DRD-8108 superó al FB-17. El híbrido FB-17 con el tratamiento LIT rindió $69,3 \mathrm{t} / \mathrm{ha}$ y $5,87 \mathrm{~kg} / \mathrm{planta}$; con CA obtuvo $75,5 \mathrm{t} / \mathrm{ha}$ y 6,39 kg/planta; y AJU alcanzó 76,4 t/ha y 6,62 kg/planta. Para el DRD-8108 con el tratamiento LIT rindió 85,4 t/ha y $8,55 \mathrm{~kg} /$ planta; CA obtuvo 86,7 t/ha y 7,45 kg/planta; y AJU alcanzó $83,9 \mathrm{t} / \mathrm{ha}$ y $7,38 \mathrm{~kg} /$ planta. La alta temperatura al interior del invernadero, fue el principal factor limitante del rendimiento alcanzado.
\end{abstract}

Palabras clave: Fertilización, hortalizas, ambiente protegido.

\begin{abstract}
Fertigation and yield of tomato hybrids growing in a greenhouse. The objective of this work was to evaluate the response of tomato hybrids to three fertilization-watering programs based on recommendations of dose compiled from the literature (LIT), curves of absorption (CA), and adjustments of information previously analized (AJU). From October 2008 to March 2009, two tomato hybrids (FB-17 and DRD-8108) were grown in the greenhouse of the Fabio Baudrit Moreno Agricultural Research Station in Alajuela, Costa Rica. No significant statistical differences were found in the commercial performance in none of the three fertilization treatments; significant differences among the hybrids were observed. The hybrid DRD-8108 surpassed FB-17. FB-17 hybrid yield for treatment LIT was $69.3 \mathrm{t} / \mathrm{ha}$ and $5.87 \mathrm{~kg} / \mathrm{plant}$; in the CA treatment was $75.5 \mathrm{t} / \mathrm{ha}$ and $6.39 \mathrm{~kg} / \mathrm{plant}$; and in the AJU treatment $76.4 \mathrm{t} / \mathrm{ha}$ and 6.62 $\mathrm{kg} /$ plant, respectively. For the DRD-8108 hybrid, the LIT treatment yield was $85.4 \mathrm{t} / \mathrm{ha}$ and $8.55 \mathrm{~kg} / \mathrm{plant}$; in the CA treatment was $86.7 \mathrm{t} / \mathrm{ha}$ and $7.45 \mathrm{~kg} / \mathrm{plant}$; finally in the AJU treatment yield was $83.9 \mathrm{t} / \mathrm{ha}$ and $7.38 \mathrm{~kg} /$ plant. The high internal greenhouse temperature was the main limiting factor that constrained yield.
\end{abstract}

Key words: Fertilization, vegetable, protected environment.

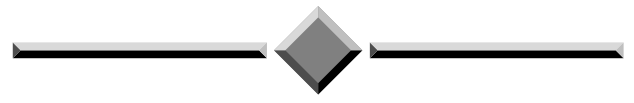

\footnotetext{
1 Recibido: 15 de diciembre, 2011. Aceptado: 30 de marzo, 2012. Este trabajo es parte de la Tesis de Maestría del primer autor. Sistema de Estudios de Posgrado. Universidad de Costa Rica.

2 Programa de Hortalizas. Estación Experimental Fabio Baudrit M., Universidad de Costa Rica. gustavo.quesada@ucr.ac.cr

3 Laboratorio de Suelos y Foliares. Centro de Investigaciones Agronómicas, Universidad de Costa Rica. floria.bertsch@ucr.ac.cr
} 


\section{INTRODUCCIÓN}

La necesidad de incrementar la producción hortícola en un contexto de escasa superficie cultivable, climas adversos y agotamiento del recurso agua, ha llevado a considerar como opción tecnológica la producción intensiva en invernaderos (Sánchez 2004). Costa Rica reporta 684 unidades de producción bajo ambiente protegido que equivalen a 687 ha de cultivos de las cuales 116 ha corresponden a solanáceas, chile y tomate principalmente (Marín 2010). Si a esta infraestructura se le combina con la fertirrigación se incrementarían los rendimientos por hectárea y la calidad de las cosechas con la posibilidad de producir constantemente durante todo el año (Bautista y Alvarado 2005).

El tomate (Lycopersicum esculentum) en Costa Rica y en el mundo es una de las hortalizas que con mayor frecuencia se siembra en invernadero (Caravaca 2008). La siembra de este cultivo es una de las más globalizadas, avanzadas e innovadoras de la industria hortícola (Costa y Heuvelink 2006). La alta demanda en los mercados y su valor comercial, además de la posibilidad de sembrar en épocas con condiciones climáticas desfavorables, justifican la importante inversión que supone el desarrollo de proyectos de este tipo bajo ambiente protegido (Cook y Calvin 2005).

Un buen manejo de la nutrición mineral es fundamental pues determina en gran medida la capacidad productiva de la planta de tomate (Snyder 2006). Por lo general muchos de los híbridos sembrados comercialmente en el país presentan buena adaptación, un excelente comportamiento y un alto potencial de rendimiento, no obstante, son muy demandantes de nutrimentos, por lo que un buen diseño y ajuste de la fertilización en tomate es fundamental (Vallejo 1999).

El fertirriego garantiza un suministro de nutrimentos directamente en el bulbo de humedecimiento, sitio donde se encuentra el mayor volumen de raíces absorbentes (Imas 2009). Esto favorece la eficiencia en el uso del agua y los fertilizantes, lo que mejora su distribución y localización. Si se emplea este recurso en forma adecuada, con el aporte de los nutrimentos que la planta demanda en el tiempo y la cantidad precisa para cada etapa fenológica, la mejora en el rendimiento alcanzado y en parámetros de calidad de la fruta (tamaño, firmeza, sanidad, sólidos solubles) es notable (Alcántar et al. 1999).
El fertirriego es una técnica exitosa; no obstante existen problemas que es necesario resolver, entre ellos la dosis de los fertilizantes y el momento de aplicación tal y como lo informan Bugarin et al. (2002). La duda surge para el productor cuando tiene ante sí diferentes programas de fertirriego para un mismo cultivo y creados con diferentes estrategias, sean estas experiencias previas (propias o de terceros), diseños a partir de curvas de absorción o recomendaciones literarias.

Este trabajo se planteó con el objetivo de evaluar el efecto de tres programas de fertirriego, sobre la producción de híbridos de tomate en invernadero.

\section{MATERIALES Y MÉTODOS}

Se evaluó el efecto de tres programas de fertirriego: Tratamiento de literatura (LIT), tratamiento de curvas de absorción (CA) y tratamiento de ajuste (AJU), sobre el comportamiento agronómico de los híbridos FB-17 (Universidad de Costa Rica) y DRD-8108 (De Ruiter Seeds). El trabajo se realizó en un invernadero multicapilla del Programa de Hortalizas de la Estación Experimental Agrícola Fabio Baudrit Moreno (EEAFBM) de la Universidad de Costa Rica, en el Barrio San José de Alajuela, a $843 \mathrm{msnm}$, a una latitud norte de $10^{\circ} 00^{\prime}$ y una longitud oeste de $84^{\circ} 15^{\prime}$.

Para el diseño de los programas de fertirriego se siguió la siguiente metodología:

\section{Literatura (LIT)}

Se recopiló información sobre requerimientos nutricionales y curvas de absorción en tomate en invernadero que reporta la literatura y aquellos sitios confiables de la red Internet (Cuadro 1).

Para definir el requerimiento final que se adoptó para el tratamiento de literatura (LIT), se determinó el promedio y la mediana a partir de las distintas fuentes consultadas (Cuadro 1). Se decidió trabajar con la mediana en lugar del promedio, porque la primera descarta los valores con mayor desviación. Para uniformar las demandas nutricionales se determinó el elemento requerido para producir una tonelada de cosecha. Esos valores se multiplicaron por 180 considerando ese valor como el rendimiento esperado 
Cuadro 1. Requerimientos nutricionales totales (en $\mathrm{kg} / \mathrm{ha}$ ) para tomate en invernadero según la literatura.

\begin{tabular}{|c|c|c|c|c|c|c|c|c|c|c|c|}
\hline \multirow{2}{*}{$\begin{array}{c}\text { Rendimiento } \\
\text { (t/ha) }\end{array}$} & \multicolumn{5}{|c|}{ Absorción según rendimiento } & \multicolumn{5}{|c|}{ Absorción para 1 tonelada } & \multirow[t]{2}{*}{ Fuente } \\
\hline & $\mathbf{N}$ & $\mathbf{P}$ & $\mathbf{K}$ & Mg & $\mathrm{Ca}$ & $\mathbf{N}$ & $\mathbf{P}$ & $\mathbf{K}$ & $\mathrm{Mg}$ & $\mathbf{C a}$ & \\
\hline 115,4 & 211 & 30 & 264 & 40 & 195 & 1,8 & 0,3 & 2,3 & 0,3 & 1,7 & Fayad et al. (2002) \\
\hline 100,0 & 200 & 44 & 500 & & & 2,0 & 0,4 & 5,0 & & & Molina (2006) \\
\hline 195,0 & 450 & 65 & 710 & & & 2,3 & 0,3 & 3,6 & & & Scaife y Bar-Yosef (1995) \\
\hline 180,0 & 562 & 110 & 886 & 72 & 139 & 3,1 & 0,6 & 4,9 & 0,4 & 0,8 & Haifa Nutri-Net (2008) \\
\hline 80,0 & 250 & 34 & 420 & 40 & 220 & 3,1 & 0,4 & 5,3 & 0,5 & 2,8 & FAO, sf. \\
\hline 1,0 & 3,25 & 0,75 & 5 & 0,35 & 0,4 & 3,3 & 0,8 & 5,0 & 0,4 & 0,4 & Moreira $(2005)^{1}$ \\
\hline 57,25 & 300 & 122 & 360 & & & 5,2 & 2,1 & 6,3 & & & Alcántar et al. (1999) \\
\hline 100,0 & 600 & 88 & 830 & & & 6,0 & 0,9 & 8,3 & & & Molina (2006) \\
\hline \multirow[t]{6}{*}{59,25} & 490 & 122 & 508 & & & 8,3 & 2,1 & 8,6 & & & Alcántar et al. (1999) \\
\hline & & & \multicolumn{3}{|c|}{ Mediana } & 3,1 & 0,6 & 5,0 & 0,4 & 1,2 & \\
\hline & & & \multicolumn{3}{|c|}{ Promedio } & 3,9 & 0,9 & 5,5 & 0,4 & 1,4 & \\
\hline & & & \multicolumn{3}{|c|}{ DE } & 2,2 & 0,7 & 2,0 & 0,1 & 1,0 & \\
\hline & & & \multicolumn{3}{|c|}{$\% \mathrm{CV}$} & $55 \%$ & $82 \%$ & $37 \%$ & $18 \%$ & $75 \%$ & \\
\hline & & & \multicolumn{3}{|c|}{$\begin{array}{l}\text { Requerimiento } \\
(180 \mathrm{t} / \mathrm{ha})\end{array}$} & 540 & 110 & 900 & 67 & 222 & \\
\hline
\end{tabular}

${ }^{1}$ Moreira, M. 2007. Requerimiento nutricional para el tomate. Alajuela, CR. Universidad de Costa Rica. Comunicación personal.

en t/ha en condiciones de invernadero tanto para el híbrido FB-17 como para el DRD-8108.

\section{Curvas de Absorción (CA)}

En un trabajo previo (Quesada y Bertsch 2009), se desarrolló la curva de absorción del híbrido FB-17 en una plantación comercial de tomate localizada en San Isidro de Heredia, Costa Rica. Cada quince días y a lo largo de todo el ciclo del cultivo se tomaron tres plantas por muestreo y se seccionaron en raíz, parte aérea (tallo, hoja y flor) y fruto. Las muestras se secaron en el laboratorio de la EEAFBM y se enviaron al Laboratorio de Suelos y Foliares (LSF) del Centro de Investigaciones Agronómicas (CIA) de la Universidad de Costa Rica, en donde se realizó la determinación de la concentración y absorción de nutrimentos. Luego se calculó el peso seco acumulado ( $\mathrm{kg} / \mathrm{ha}$ ) por tejido. Relacionando ambas informaciones se estimó la absorción por la planta de los elementos N, P, K, Ca, Mg y $\mathrm{S}$; además se calculó la extracción de los nutrimentos considerando una densidad de siembra de 19230 plantas/ha y un rendimiento estimado de 162 toneladas de fruto por hectárea. Cada nutrimento se graficó colocando los $\mathrm{kg}$ de nutriente por tejido/ha contra el tiempo. El tratamiento de curva de absorción (CA) consistió en el seguimiento riguroso de esta curva de absorción generada.

En el caso del híbrido de tomate DRD-8108 no se realizó una curva de absorción de nutrimentos como tal, pero se obtuvo la información a partir del trabajo de Alfaro $(2007)^{4}$ que elaboró un programa de fertirriego para este híbrido basado en requisitos de extracción del tomate en diferentes fases fenológicas del cultivo. Esto permitió definir los requerimientos nutricionales de ese híbrido por etapa; se consideró como óptima la distribución porcentual de los nutrimentos que se obtuvo a partir de la curva de absorción del híbrido FB-17, por lo que se siguió el mismo patrón de distribución porcentual en el híbrido DRD-8108. En el Cuadro 2 se resumen los requerimientos de absorción de ambos híbridos.

4 Alfaro, M. 2007. Extracción de nutrientes del híbrido de tomate DRD-8108. San José, CR. Universidad de Costa Rica. Comunicación personal. 
Cuadro 2. Requerimientos de absorción (en $\mathrm{kg} / \mathrm{ha}$ ) de dos híbridos de tomate FB-17 y DRD-8108 en el tratamiento de curvas de absorción. Quesada y Bertsch 2009.

\begin{tabular}{lccccc}
\hline & \multicolumn{5}{c}{ Absorción (180 t/ha) } \\
\cline { 2 - 6 } Híbrido & N & P & K & Mg & Ca \\
\hline FB-17 & 580 & 43 & 846 & 104 & 405 \\
DRD-8108 & 562 & 40 & 863 & 67 & 347 \\
\hline
\end{tabular}

\section{Ajuste (AJU)}

El tratamiento de ajuste (AJU) se planteó para poder manejar con flexibilidad cambios en la estrategia de fertilización de acuerdo con el comportamiento en campo que se iba observando en la planta. Práctica común del productor es el realizar ajustes en la fertilización a través de las diferentes etapas fenológicas del cultivo para potenciar el desarrollo de la planta y/o corregir deficiencias nutricionales. Para generar este tratamiento se consideró tanto la información recopilada en la literatura (LIT) como la que se obtuvo a partir de la curva de absorción elaborada (CA). Para cada elemento se incluyeron criterios específicos y se decidió utilizar una única cantidad para ambos híbridos. Como punto de partida se promediaron los requerimientos de los tratamientos LIT y CA (híbrido FB-17) para definir la cantidad de nutrimentos a suministrar en este tratamiento para los dos híbridos en estudio (Cuadro 3). En la Figura 1 ( $\mathrm{y}$ y b) se muestra en términos porcentuales

Cuadro 3. Requerimientos de absorción (en $\mathrm{kg} / \mathrm{ha}$ ) para el cultivo de tomate en el tratamiento de ajuste (promedio de los requerimientos según literatura y según curva de absorción del híbrido de tomate FB-17) estimando un rendimiento de cosecha de $180 \mathrm{t} / \mathrm{ha}$.

\begin{tabular}{lccccc}
\hline & \multicolumn{5}{c}{ Absorción (180 t/ha) } \\
\cline { 2 - 6 } Tratamiento & $\mathbf{N}$ & $\mathbf{P}$ & $\mathbf{K}$ & $\mathbf{M g}$ & $\mathbf{C a}$ \\
\hline LIT & 540 & 110 & 900 & 67 & 222 \\
CA (FB-17) & 580 & 43 & 846 & 104 & 405 \\
\cline { 2 - 6 }$\quad$ promedio: & 560 & 77 & 873 & 86 & 313 \\
& & & & & \\
AJU & 560 & 77 & 873 & 86 & 313 \\
\hline
\end{tabular}

LIT $=$ Tratamiento de literatura; $C A=$ Tratamiento de curvas de absorción; AJU= Tratamiento de ajuste.
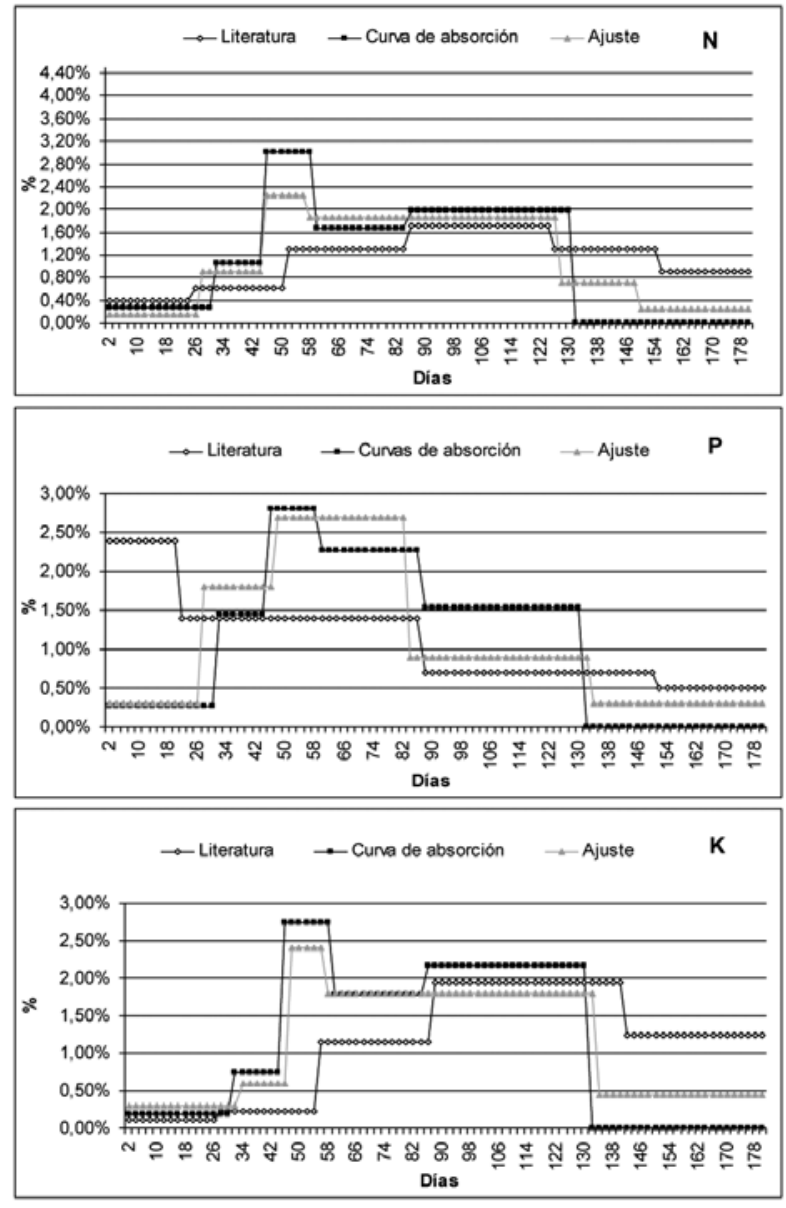

Figura 1a. Distribución diaria porcentual del suministro de N, $\mathrm{P}$ y K en tres tratamientos de fertilización durante un ciclo de cultivo del tomate a los 180 días en condiciones de invernadero. Alajuela, Costa Rica. 2009.

la manera como finalmente fue distribuido, a lo largo del ciclo, el requerimiento de los elementos $\mathrm{N}, \mathrm{P}, \mathrm{K}$, $\mathrm{Ca}$ y $\mathrm{Mg}$ a lo largo del ciclo en los tres tratamientos.

Definidas las estrategias de fertilización, la fase de invernadero se desarrolló en el periodo comprendido del 30 de setiembre del 2008 (fecha del trasplante) al 13 de abril del 2009. La distancia entre plantas fue de $0,25 \mathrm{~m}$ en el sistema "tres bolillo" y entre hileras de $1,6 \mathrm{~m}$. Bajo este sistema se manejaron 2,5 ejes productivos por metro cuadrado.

La siembra se realizó directamente sobre el suelo del invernadero, previa desinfección con Butrol 31,5 EC (TCMTB, fungicida-bactericida de amplio 

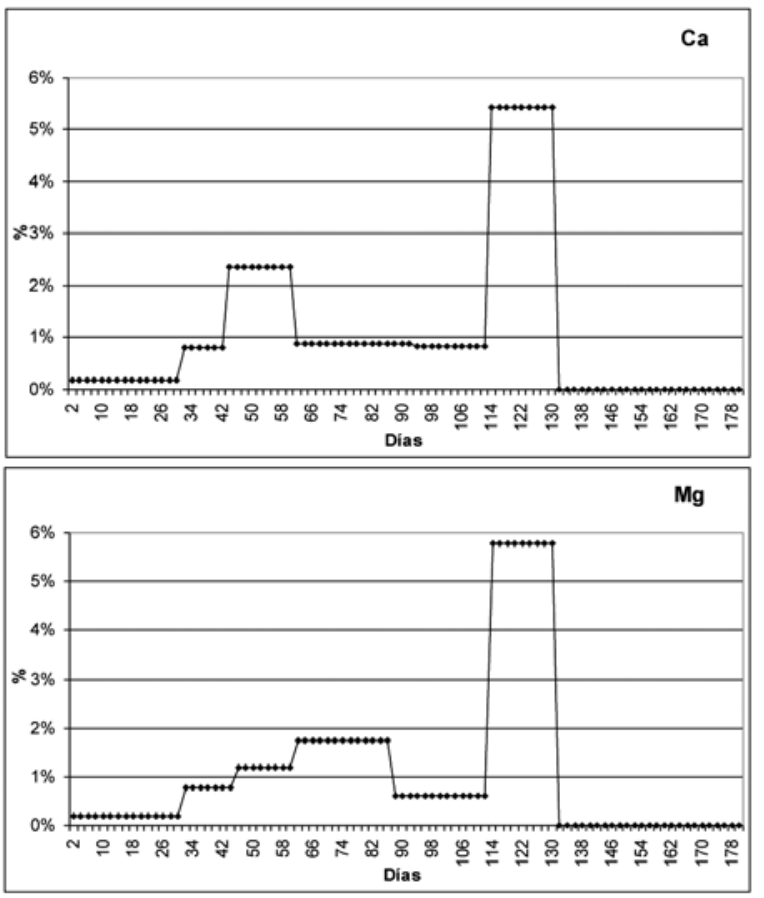

Figura 1b. Distribución diaria porcentual del suministro de $\mathrm{Ca}$ y $\mathrm{Mg}$ durante un ciclo de cultivo de tomate de 180 días en condiciones de invernadero. Alajuela, Costa Rica. 2009. (misma distribución porcentual para los tres tratamientos de fertilización).

espectro) a una concentración de $1 \mathrm{ml} / \mathrm{m}^{2}$. El suelo del invernadero de la EEAFBM es un inceptisol clasificado taxonómicamente como Typic "Andic" Plagganthrepts, con características de andisol en los horizontes inferiores. El análisis químico de este suelo se reporta en el Cuadro 4.

El manejo agronómico empleado es el que convencionalmente se utiliza en el Programa de Hortalizas de la EEAFBM en condiciones de invernadero. Este se basó en el mantenimiento a un eje de la planta, con deshijas y deshojas continuas realizadas semanalmente. Para las labores de fitoprotección se aplicaron insecticidas (Acefato, Imidacloprid, Endosulfan, Abamectina; Deltametrina, Tiametoxan) y fungicidas (Captan, Metalaxil, Policúpricos + Mancozeb, Triadimefon, Azoxistrobina, Azufre) según fue necesario, en rotación y con productos específicos autorizados para tomate, cumpliendo con las normativas sobre BPA. El tutorado se hizo enredando el tallo de la planta al hilo de conducción aéreo; se siguió la práctica de cuelgue y descuelgue para manejar adecuadamente la altura de la planta y facilitar las labores de cultivo. Se hicieron aplicaciones foliares de microelementos ( $\mathrm{B}, \mathrm{Zn}, \mathrm{Fe}, \mathrm{Mn}, \mathrm{Cu}, \mathrm{Mo}$ ) quincenalmente, igual para todos los tratamientos.

El riego se realizó en forma diaria conforme a los requerimientos de la planta $(\mathrm{Kc})$ y a las condiciones del ambiente (Cuadro 5); se empleó un sistema presurizado utilizando poliducto, pajillas y goteros. La descarga promedio de los goteros fue de $2,26 \mathrm{~L} / \mathrm{h}$ y el coeficiente de uniformidad del sistema estuvo cercano al $93 \%$.

Los nutrimentos se suministraron mediante el sistema de riego; cada dos días durante 180 días para los tratamientos LIT y AJU y cada dos días durante 130 días para el tratamiento CA. En todos los casos se preparó la solución concentrada de fertilizante correspondiente a la demanda nutricional específica para ese día de acuerdo con el ciclo fenológico del cultivo y el tratamiento respectivo. Para evitar problemas por incompatibilidad de fertilizantes, se manejaron dos soluciones; una conformada por los nitratos de calcio, potasio y amonio, y cloruros de calcio y potasio. La otra solución contenía fosfato monoamónico y sulfato de magnesio. Para la aplicación del fertirriego se utilizó un inyector dosificador hidraúlico (Dosatron) para garantizar una distribución uniforme del fertilizante. Mediante llaves de paso manuales colocadas al inicio del poliducto en cada hilera de cultivo, se controló que

Cuadro 4. Análisis químico del suelo del invernadero de la Estación Experimental Fabio Baudrit Moreno, Alajuela, Costa Rica. 2008.

\begin{tabular}{|c|c|c|c|c|c|c|c|c|c|c|c|}
\hline pH & & & $\operatorname{col}(+) /$ & & & $\%$ & & & $\mathrm{mg} / \mathrm{l}$ & & \\
\hline $\mathrm{H}_{2} \mathrm{O}$ & Acidez & $\mathrm{Ca}$ & $\mathrm{Mg}$ & $\mathrm{K}$ & CICE & SA & $\mathrm{P}$ & $\mathrm{Zn}$ & $\mathrm{Cu}$ & $\mathrm{Fe}$ & $\mathrm{Mn}$ \\
\hline$<5,5$ & $>0,5$ & $<4$ & $<1$ & $<0,2$ & $<5$ & $>10$ & $<10$ & $<3$ & $<1$ & $<10$ & $<5$ \\
\hline 5,4 & 0,33 & 10,06 & 2,23 & 0,5 & 13,11 & $2 \%$ & 11 & 5,4 & 28 & 238 & 22 \\
\hline
\end{tabular}

Acidez, $\mathrm{Ca}$ y $\mathrm{Mg}$ en $\mathrm{KCl}$, resto en solución extractora Olsen Modificada. 
Cuadro 5. Promedios mensuales de temperatura, humedad relativa y radiación dentro del invernadero de la Estación Experimental Fabio Baudrit Moreno, Alajuela, Costa Rica. 2009.

\begin{tabular}{lccccccc}
\hline Mes-año & \multicolumn{3}{c}{ Temperatura del aire $\left({ }^{\circ} \mathbf{C}\right)$} & \multicolumn{2}{c}{ Humedad relativa del aire (\%) } & \multicolumn{2}{c}{ Radiación PAR } \\
\cline { 2 - 8 } & Máxima & Mínima & Promedio & Máxima & Mínima & Promedio & $\begin{array}{c}\text { Máxima } \\
\left(\boldsymbol{\mu} \mathbf{M} / \mathbf{m}^{2} \mathbf{s}\right)\end{array}$ \\
\hline sep-08 & $29,17 \pm 1,84$ & $23,43 \pm 0,23$ & $25,74 \pm 0,78$ & $69,10 \pm 1,96$ & $44,38 \pm 29,74$ & $53,38 \pm 25,49$ & - \\
oct-08 & $34,12 \pm 2,06$ & $18,70 \pm 0,81$ & $22,92 \pm 2,55$ & $93,42 \pm 5,88$ & $35,50 \pm 9,11$ & $72,81 \pm 7,49$ & $1102,75 \pm 262,30$ \\
nov-08 & $32,54 \pm 2,44$ & $18,06 \pm 0,92$ & $22,98 \pm 0,74$ & $94,05 \pm 7,58$ & $40,26 \pm 11,44$ & $74,60 \pm 9,47$ & $969,69 \pm 250,77$ \\
dic-08 & $34,14 \pm 1,79$ & $16,96 \pm 1,41$ & $23,06 \pm 0,71$ & $92,95 \pm 5,50$ & $29,73 \pm 7,52$ & $66,95 \pm 7,16$ & $909,54 \pm 142,75$ \\
ene-09 & $33,10 \pm 3,06$ & $16,27 \pm 4,46$ & $22,07 \pm 4,52$ & $86,09 \pm 6,69$ & $29,81 \pm 9,94$ & $60,35 \pm 14,17$ & $865,74 \pm 195,07$ \\
feb-09 & $34,76 \pm 1,59$ & $17,48 \pm 1,52$ & $23,75 \pm 0,65$ & $80,41 \pm 6,26$ & $27,46 \pm 6,70$ & $57,68 \pm 5,43$ & $987,79 \pm 99,74$ \\
mar-09 & $34,30 \pm 1,16$ & $16,40 \pm 5,52$ & $22,86 \pm 3,57$ & $82,30 \pm 3,58$ & $28,20 \pm 4,79$ & $57,93 \pm 2,88$ & $1104,74 \pm 63,15$ \\
\hline
\end{tabular}

la solución nutritiva de un tratamiento no se aplicara en un tratamiento que no fuera el correspondiente. Entre cada tratamiento se dio un tiempo de riego de un minuto para efectuar el lavado de la tubería y evitar el traslape de soluciones nutritivas. Los tiempos de duración del fertirriego se definieron para poder suministrar toda la solución nutritiva correspondiente y que además se ajustara a la necesidad hídrica del cultivo.

El diseño experimental utilizado fue un bifactorial $(2 \times 3)$ con arreglo en parcelas divididas y cuatro repeticiones. La parcela principal (factor A) fue el tratamiento de fertilización y la subparcela (factor B) el híbrido. Cada repetición estuvo conformada por 216 plantas para un total de 864 plantas. El área total del ensayo fue de $345 \mathrm{~m}^{2}$. La unidad experimental fue una parcela de $28,8 \mathrm{~m}^{2}$ que estuvo conformada por 36 plantas de cada uno de los híbridos para un total de 72 plantas por unidad experimental. En total se trabajó con 23 grados de libertad.

Para cada uno de los tratamientos y de los híbridos en evaluación, durante veintiocho cosechas, se cuantificó el rendimiento comercial, dado por el número y peso de frutos de primera ( $\geq 200 \mathrm{~g}$ por fruto), segunda ( $\geq 120$ a $200 \mathrm{~g}$ por fruto) y tercera categoría $(<120 \mathrm{~g}$ por fruto), además de la categoría de rechazo (deformaciones, pudrición apical, estrellado y enfermo). Para el análisis estadístico de los datos se realizó un análisis de varianza; la prueba de comparación de medias de los tratamientos se realizó mediante la prueba LSD Fisher. Para ello se emplearon los paquetes estadísticos InfoStat 2002 y Statistica 2001.

\section{RESULTADOS Y DISCUSIÓN}

Las diferencias entre los tratamientos no sólo se atribuyen al aporte en sí de cada uno de los elementos (cantidad) sino también al momento del ciclo fenológico del cultivo en que se suministraron (Figura 1a, 1b). En relación con la cantidad, el tratamiento $\mathrm{CA}$ fue el de mayor aporte de $\mathrm{N}$, Ca y Mg, mientras que el tratamiento LIT demandó más P y K. El consumo del tratamiento AJU siempre estuvo entre los otros dos tratamientos, aunque para el $\mathrm{N}$ y $\mathrm{K}$ la demanda fue más cercana al LIT, mientras que para el $\mathrm{P}, \mathrm{Ca}$ y $\mathrm{Mg}$ la demanda fue intermedia a la de los tratamientos LIT y CA.

Si se analiza desde el punto de vista de la distribución porcentual, también son notables las diferencias entre tratamientos para los elementos $\mathrm{N}, \mathrm{P}$ y K puesto que hay una alternancia clara a lo largo del ciclo relacionada con el aporte porcentual de los distintos tratamientos; sin embargo se apreció una tendencia de un mayor aporte porcentual del tratamiento LIT al inicio y al final del ciclo, mientras que al intermedio del ciclo, predominó la demanda del tratamiento CA. Sólo en pocas ocasiones el tratamiento AJU fue el de mayor fertilización porcentual.

\section{Rendimiento comercial categorías primera, segun- da y tercera}

El rendimiento en el cultivo de tomate está dado por el número y peso del fruto en sus distintas categorías, sin embargo, la retribución económica está determinada 
por el peso. Las frutas se clasifican en primera, segunda y tercera, según los criterios de peso establecidos por el mercado (Caravaca 2008). Si bien esta categorización está en función del peso de la fruta, no descarta aspectos de calidad (forma, apariencia, firmeza) y sanidad de la cosecha (Cerdas y Montero 2002).

\section{Número de frutos}

El número de frutos comerciales y su distribución para los híbridos FB-17 y DRD-8108 en las categorías de mayor importancia económica, se presentan en el Cuadro 6 y la Figura 2.

Hay una marcada diferencia estadística en el número de frutos de primera y segunda categoría (Figura 2), que favorece al híbrido DRD-8108 sobre el FB-17. Sin embargo, no hay un efecto de los tres planes de fertilización evaluados sobre la cantidad de frutos en ninguno de los híbridos.

El material DRD-8108 produjo mayor porcentaje de frutos de primera categoría al compararlo con el híbrido FB-17; este último tuvo más de $60 \%$ de fruta de tercera categoría en los tres tratamientos, que es la que menor retribución económica genera. Pese a esto el híbrido FB-17 produjo estadísticamente más número de frutos totales que el híbrido DRD-8108, aunque el número de frutos por planta solo diferencia el tratamiento AJU del híbrido FB-17 del tratamiento CA del híbrido DRD-8108.

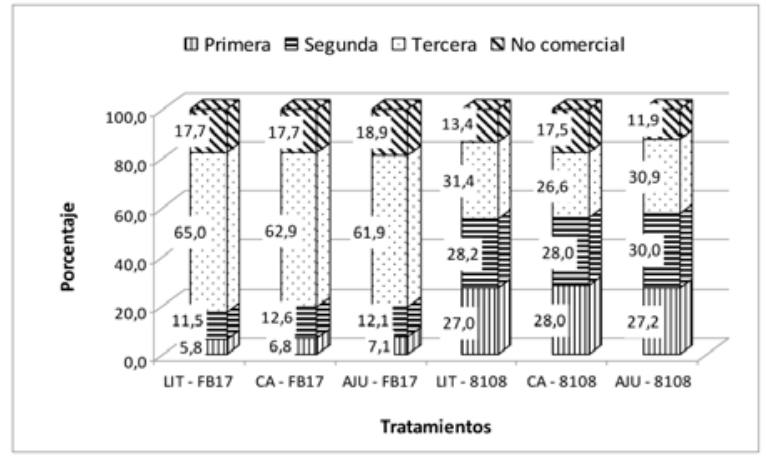

Figura 2. Distribución porcentual de frutos en las categorías comercial y no comercial, en híbridos de tomate en invernadero sometidos a distintos planes de fertilización durante un ciclo completo de cultivo. Estación Experimental Fabio Baudrit Moreno, Alajuela, Costa Rica. 2009.

\section{Peso $(\mathrm{kg})$ de frutos}

El peso y la distribución porcentual de frutos de tomate en sus categorías comerciales se muestran en el Cuadro 7 y Figura 3.

Nuevamente el híbrido DRD-8108 presentó mayor peso en las categorías primera y segunda y el híbrido FB-17 en la de tercera categoría. No existieron diferencias estadísticas significativas en los tres planes de fertilización evaluados. Se destaca que poco más

Cuadro 6. Número de frutos totales y por categoría comercial en híbridos de tomate en invernadero sometidos a distintos planes de fertilización durante un ciclo completo de cultivo. Alajuela, Costa Rica. 2009.

\begin{tabular}{lccccc}
\hline Tratamiento/Híbrido & Primera & Segunda & Tercera & Total $^{1 /}$ & Fruto/planta $^{-128 \mathrm{~b}}$ \\
\hline LIT - FB-17 & $115 \mathrm{~b}$ & $228 \mathrm{~b}$ & $1287 \mathrm{a}$ & $1980 \mathrm{a}$ & $56,6 \mathrm{ab}$ \\
CA - FB-17 & $137 \mathrm{~b}$ & $255 \mathrm{~b}$ & $1269 \mathrm{a}$ & $2019 \mathrm{a}$ & $57,7 \mathrm{ab}$ \\
AJU - FB-17 & $145 \mathrm{~b}$ & $246 \mathrm{~b}$ & $1260 \mathrm{a}$ & $2034 \mathrm{a}$ & $58,1 \mathrm{a}$ \\
LIT - DRD-8108 & $390 \mathrm{a}$ & $408 \mathrm{a}$ & $455 \mathrm{~b}$ & $1447 \mathrm{~b}$ & $41,3 \mathrm{ab}$ \\
CA - DRD-8108 & $400 \mathrm{a}$ & $399 \mathrm{a}$ & $379 \mathrm{~b}$ & $1427 \mathrm{~b}$ & $40,8 \mathrm{~b}$ \\
AJU - DRD-8108 & $392 \mathrm{a}$ & $432 \mathrm{a}$ & $444 \mathrm{~b}$ & $1440 \mathrm{~b}$ & $41,1 \mathrm{ab}$ \\
\hline
\end{tabular}

${ }^{1 /}$ datos incluyen fruta de las categorías comercial y rechazo.

$\mathrm{a}, \mathrm{b}$ medias con igual letra en la misma columna no difieren estadísticamente según prueba de LSD Fisher $(\mathrm{P}<$ $0,05)$.

LIT= Tratamiento de literatura; $\mathrm{CA}=$ Tratamiento de curvas de absorción; AJU= Tratamiento de ajuste . 
Cuadro 7. Peso $(\mathrm{kg})$ de los frutos totales y por categoría comercial en híbridos de tomate en invernadero sometidos a distintos planes de fertilización durante un ciclo completo de cultivo. Estación Experimental Fabio Baudrit Moreno, Alajuela, Costa Rica. 2009.

\begin{tabular}{lccccc}
\hline Tratamiento/Híbrido & Primera & Segunda & Tercera & Total $^{1 /}$ & Frutos/planta $^{\text {1 }}$ \\
\hline LIT - FB-17 & $29,8 \mathrm{~b}$ & $38,9 \mathrm{~b}$ & $86,5 \mathrm{a}$ & $199,6 \mathrm{~b}$ & $5,87 \mathrm{~b}$ \\
CA - FB-17 & $35,3 \mathrm{~b}$ & $44,4 \mathrm{~b}$ & $90,0 \mathrm{a}$ & $217,4 \mathrm{ab}$ & $6,39 \mathrm{~b}$ \\
AJU - FB-17 & $37,7 \mathrm{~b}$ & $43,3 \mathrm{~b}$ & $85,8 \mathrm{a}$ & $220,1 \mathrm{ab}$ & $6,62 \mathrm{~b}$ \\
LIT - DRD-8108 & $102,7 \mathrm{a}$ & $70,7 \mathrm{a}$ & $36,5 \mathrm{~b}$ & $245,9 \mathrm{a}$ & $8,55 \mathrm{a}$ \\
CA - DRD-8108 & $105,3 \mathrm{a}$ & $69,9 \mathrm{a}$ & $30,8 \mathrm{~b}$ & $249,7 \mathrm{a}$ & $7,45 \mathrm{ab}$ \\
AJU - DRD-8108 & $101,7 \mathrm{a}$ & $71,7 \mathrm{a}$ & $36,8 \mathrm{~b}$ & $241,7 \mathrm{a}$ & $7,38 \mathrm{ab}$ \\
\hline
\end{tabular}

${ }^{1 /}$ datos incluyen fruta de las categorías comercial y no comercial.

a, b medias con igual letra en la misma columna no difieren estadísticamente según prueba de LSD Fisher $(\mathrm{P}<0,05)$.

LIT $=$ Tratamiento de literatura; $\mathrm{CA}=$ Tratamiento de curvas de absorción; $\mathrm{AJU}=$ Tratamiento de ajuste.

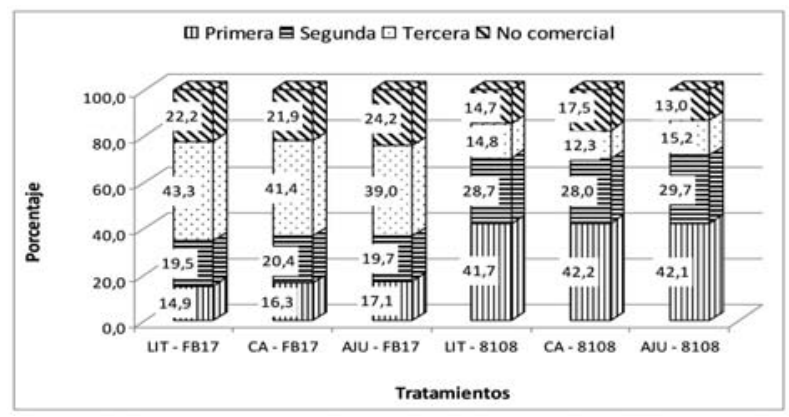

Figura 3. Distribución porcentual del peso de frutos en las categorías comercial y no comercial, en híbridos de tomate en invernadero sometidos a distintos planes de fertilización durante un ciclo completo de cultivo. Estación Experimental Fabio Baudrit Moreno, Alajuela, Costa Rica. 2009.

del $40 \%$ del total del peso de la fruta en el híbrido DRD-8108 corresponde al de primera categoría, lo que contrasta con el híbrido FB-17 que para esta misma categoría sólo alcanzó un porcentaje cercano al 15\%. En el peso total de todos los tratamientos, no hubo diferencia estadística salvo para el tratamiento LIT del híbrido FB-17 que mostró el peso total más bajo.

Aún cuando se utilizaron diferentes dosis y épocas de aplicación del fertilizante, desde el punto de vista del rendimiento comercial (número y peso de frutos), los planes de fertilización no mostraron diferencias estadísticas entre tratamientos. Una de las principales razones pudo ser la capacidad de amortiguamiento o poder "buffer" del suelo, que neutraliza los efectos de las distintas dosis y momentos de aplicación de los nutrimentos, al sucederse la interacción de los procesos de solubilización, fijación e intercambio iónico que se dan en el complejo coloidal del suelo (Bertsch 1998). Este efecto amortiguador puede acentuarse en un suelo bajo condiciones de invernadero, dado que en este caso el sistema suelo es más estable al no verse afectado por factores modificadores como el clima, escorrentías, lixiviación o la restricción de la participación de la fracción orgánica entre otros.

Es probable que si esta investigación se hubiera desarrollado utilizando sustratos inertes como medio de cultivo en lugar de la siembra directa en el suelo mineral, se hubiera observado mayor diferenciación de los planes de fertilización. Esto porque los sustratos, al no poseer un complejo coloidal como tal, presentan una capacidad de intercambio catiónico más reducida (Handreck y Black 2002), proceso de interacción de nutrimentos entre las fases sólidas y líquidas que define en buena medida el aprovechamiento de los elementos requeridos para el establecimiento y óptimo desarrollo de los cultivos (Alcántar y Trejo 2007). Otros trabajos como los que reportaron Araujo et al. (2007) y Marín et al. (2008) en experimentos similares, no mostraron diferencias significativas en el rendimiento comercial de tomate obtenido, a partir de programas diferentes de fertirriego. 
Debe considerarse que la elaboración de una curva de absorción implica tiempo y un costo económico considerable, pero la información que se recaba de ella es de suma importancia. Como muestra, a partir de lo indicado por la curva de absorción, se decidió no fertilizar después de los 130 dds, y pese a que faltaban 50 días aún para llegar al final del ciclo del cultivo del tomate, el rendimiento no decayó y se mantuvo similar al de los tratamientos que continuaron suministrando fertilizante. Esto indica que incurrir en una fertilización hacia el final del ciclo de cultivo puede ser inefectivo, pues no sólo la planta no hace aprovechamiento de los nutrimentos disponibles, sino que también se invierten recursos innecesarios como en mano de obra, las sales fertilizantes solubles, la preparación de la solución nutritiva y sistema de fertirriego. Fertilizar de más implica por ende mayor costo económico y también un impacto sobre el ambiente por riesgos de contaminación. Es evidente que maximizar la eficiencia de la fertilización conociendo los momentos de máxima absorción de nutrimentos de un cultivo es fundamental en aquellos de ciclo corto y fertilización intensiva (Bertsch 2003), como ocurre en la mayoría de hortalizas producidas en condiciones de invernadero.

Respecto al efecto de los híbridos es claro que en las categorías de mayor importancia económica (frutos de primera y segunda) el material DRD-8108 superó notablemente al FB-17. Debe indicarse que el híbrido DRD-8108 es un material consolidado y está dentro de los cultivares de tomate que con mayor frecuencia se siembran en el país (Caravaca 2008); esto debido a su buena adaptación agroecológica, resistencia a varias enfermedades y alto potencial de rendimiento (hasta $10 \mathrm{~kg}$ de frutos por planta).

Fue notoria la gran cantidad de fruta de tercera categoría en el híbrido FB-17 y su bajo rendimiento. Si bien este material aún está en fase experimental, otras experiencias realizadas con anterioridad en fincas comerciales habían arrojado en todos los casos rendimientos superiores a 100 t/ha. Además en la elaboración previa de la curva de absorción no se presentó ningún inconveniente y se alcanzó un rendimiento de $162 \mathrm{t} / \mathrm{ha}$. Sin embargo, un problema en un lote de semilla híbrida proveniente de una línea segregante afectó la productividad de este material, lo que se reflejó en las categorías de importancia económica. Aún así la prolificidad del material se mantuvo al observarse un número total de frutos estadísticamente superior al híbrido DRD-8108 en los tres programas de fertilización implementados (Cuadro 6).

Si bien el rendimiento supera el promedio nacional de producción de tomate a campo abierto que reporta SEPSA (2011) y que se sitúa entre 65 a 70 t/ha en los últimos años (Cuadro 8), no llega a alcanzar el mínimo esperado para invernadero que es de $100 \mathrm{t} / \mathrm{ha}$, ni mucho menos el promedio nacional para invernadero que es próximo a 150 t/ha (Caravaca 2008).

Cuadro 8. Rendimiento (t/ha) de dos híbridos de tomate en invernadero sometidos a tres planes de fertilización durante un ciclo completo de cultivo. Estación Experimental Fabio Baudrit Moreno, Alajuela, Costa Rica. 2009.

\begin{tabular}{lc}
\hline Tratamiento & Rendimiento (t/ha) \\
\hline LIT - FB-17 & 69,3 \\
CA - FB-17 & 75,5 \\
AJU - FB-17 & 76,4 \\
LIT - DRD-8108 & 85,4 \\
CA - DRD-8108 & 86,7 \\
AJU - DRD-8108 & 83,9 \\
\hline
\end{tabular}

LIT $=$ Tratamiento de literatura; $\mathrm{CA}=$ Tratamiento de curvas de absorción; AJU= Tratamiento de ajuste.

Varios factores influyeron para no poder lograr un rendimiento superior. El más determinante de ellos fue la temperatura que se registró al interior del invernadero (Cuadro 5). Según Gómez et al. (2000), los procesos esenciales para una óptima fructificación son la producción de polen viable, la transferencia de polen al estigma, la germinación del grano de polen, el crecimiento del tubo polínico y la unión del gameto masculino con el óvulo viable, todos procesos termosensibles.

La alta radiación típica en la zona, con un promedio de $990 \mu \mathrm{M} / \mathrm{m}^{2}$ s y temperaturas máximas cercanas a 33$34^{\circ} \mathrm{C}$ en el interior del invernadero de la EEAFBM, afectaron severamente la producción. Nicola et al. (2009) informan que la temperatura óptima para un desarrollo vigoroso del cultivo de tomate y una buena fructificación varía entre 20 y $27^{\circ} \mathrm{C}$. Naika et al. citados por Nicola et al. (2009), indican que temperaturas nocturnas inferiores 
a $18^{\circ} \mathrm{C}$ pueden provocar aborto de frutos, lo que en algún momento se presentó en el invernadero especialmente en los meses de diciembre, enero, febrero y marzo, con mínimas que variaron entre 16 y $17^{\circ} \mathrm{C}$.

Ligado al aumento de la temperatura en un invernadero está la reducción de la humedad relativa. Idealmente, el invernadero debería mantenerse a una humedad relativa de 60 a 70\% (Snyder 2006) o al menos cercana a $50 \%$. Sin embargo en el invernadero de la EEAFBM la humedad relativa registraba con frecuencia valores por debajo del $30 \%$, especialmente en los meses de la época seca (Cuadro 5); la falta de equipamiento del invernadero (pantalla térmica, destratificadores de aire, micro-nebulizadores independientes del sistema de riego principal) impide una correcta regulación del clima interno. Esto favorece el desecamiento de la hoja de la planta, el cierre estomático y se afecta la producción. A pesar del uso de un sistema de micro-nebulización (fogg system), no se logró modificar favorablemente ni la temperatura ni la humedad relativa al interior del invernadero. Lo anterior debido a que la presión del sistema de riego no fue lo suficientemente elevada para lograr una gota realmente fina que lograra el objetivo de reducir el calor y enfriar el invernadero.

Una limitante adicional que desfavoreció el rendimiento obtenido fue la dificultad para realizar una efectiva polinización. Ocasionalmente con movimientos manuales en el sistema de tutorado se promovía la polinización, sin embargo esta práctica no es tan eficiente como la que pueden realizar agentes polinizadores o el uso de vibradores eléctricos.

$\mathrm{Al}$ no alcanzarse los niveles de producción estimados, la fertilización efectuada bajo cualquiera de los sistemas resultó abundante y no se llegaron a producir situaciones limitantes que pudieran haber expresado diferencias más claras en el rendimiento.

\section{Frutos no comerciales}

La retribución económica de la actividad tomatera en invernaderos de alta tecnología se restringe cuando un porcentaje mayor al $5 \%$ del total de la producción cae dentro de la categoría rechazo ${ }^{5}$; es el caso de frutos con pudrición apical, deformación y fruta estrellada (Cuadro 9).

\footnotetext{
5 Marín, F. 2012. Exigencias de calidad en mercados de tomate. San José, CR. Programa Nacional de Ambientes Protegidos. Comunicación personal.
}

Cuadro 9. Número de frutos no comerciales en dos híbridos de tomate en invernadero sometidos a tres planes de fertilización durante un ciclo completo de cultivo. Estación Experimental Fabio Baudrit Moreno, Alajuela, Costa Rica. 2009.

\begin{tabular}{lccc|}
\hline Tratamiento & Deforme & Pudr. apical & Estrellado \\
\hline i - FB-17 & $80 \mathrm{a}^{1 /}$ & $160 \mathrm{a}$ & $109 \mathrm{a}$ \\
ii - FB-17 & $95 \mathrm{a}$ & $150 \mathrm{a}$ & $109 \mathrm{a}$ \\
iii - FB-17 & $98 \mathrm{a}$ & $165 \mathrm{a}$ & $115 \mathrm{a}$ \\
i - DRD-8108 & $40 \mathrm{~b}$ & $41 \mathrm{~b}$ & $107 \mathrm{a}$ \\
ii - DRD-8108 & $34 \mathrm{~b}$ & $76 \mathrm{~b}$ & $135 \mathrm{a}$ \\
iii - DRD-8108 & $34 \mathrm{~b}$ & $51 \mathrm{~b}$ & $81 \mathrm{a}$ \\
\hline
\end{tabular}

${ }^{1 /}$ medias con igual letra en la misma columna no difieren estadísticamente según prueba de LSD Fisher $(\mathrm{P}<0,05)$.

La pudrición apical fue la principal causa de rechazo de los frutos no comerciales. Este es un problema muy común en solanáceas como chile y tomate y es resultado de la falta de calcio en el ápice de la fruta. Esto no necesariamente es indicador de deficiencia del elemento, ya que también la alta tasa de transpiración de la planta producto de las elevadas temperaturas en el interior del invernadero dificultó la asimilación del $\mathrm{Ca}$ en el fruto. No se presentaron diferencias entre los tratamientos de fertilización pero sí en los híbridos, donde el FB-17 mostró mayor susceptibilidad, especialmente hacia el final del ciclo del cultivo con una planta metabólicamente más desgastada. En todo caso, de acuerdo con los niveles de extracción de ambos híbridos, el FB-17 es mayor extractor de Ca (405 contra $347 \mathrm{~kg} / \mathrm{ha}$ ) que el DRD-8108.

En relación con la deformación de frutos de tomate, la mayoría de las veces se debe a problemas de mala polinización al momento de la antesis de la flor, así como al desarrollo no uniforme de la semilla con el fruto (Snyder 2006). La polinización pudo ser insuficiente, pues las altas temperaturas favorecen la esterilidad del polen (Gómez et al. 2000). Es así como en el invernadero se observaron frutos con polinización parcial de los lóculos, dando como resultado desarrollo desuniforme en la baya. El híbrido de tomate FB-17 fue el material con más problemas en este sentido; la pérdida de calidad de este cultivar producto de la segregación genética que lo afectó lo hizo más sensible a problemas de 
polinización. No se observaron diferencias estadísticas significativas entre los tratamientos de fertilización, debido a que la condición climática (particularmente la temperatura) determinó en mayor medida la eficiencia de la polinización por sobre la concentración de nutrimentos en el medio.

La fruta con estrella es aquella que muestra rajaduras en esa forma en la sección próxima al pedúnculo. La aparición de fruta estrellada es favorecida también por la alta temperatura en el invernadero. En este caso, el exocarpo de la fruta alcanza temperaturas más altas que el mesocarpo y el endocarpo; esto causó la ruptura de células adyacentes mostrándose la piel separada o reventada, debido a la imposibilidad de esos tejidos de expandirse y contraerse al cambiar la temperatura (Snyder 2006). No se presentó ninguna diferencia estadística en la incidencia de fruta con estrella, ni en los tratamientos ni en los híbridos, lo que indica que este daño es atribuible a causas abióticas, temperatura particularmente y la fertilización no tuvo influencia alguna.

\section{LITERATURA CITADA}

Alcántar, G; Villareal, M; Aguilar, A. 1999. Tomato growth (Lycopersicon esculentun Mill), and nutrient utilization in response to varying fertigation programs. Proc. Int. Sym. Growing Media and Hydroponics. Acta Horticulturae 481:385-391.

Alcántar, G; Trejo, L. 2007. Nutrición de cultivos. Ediciones Mundi Prensa. Colegio de Postgraduados. México DF, México. 438 p.

Araujo, C; Rezende, PC; Sigueyuki, C; Bernardes, M. 2007. Critérios para a determinação da dose de nitrogênio a ser aplicada no tomateiro em ambiente protegido. Horticultura Brasileira 25:327-332.

Bautista, N; Alvarado, J. 2005. Producción de jitomate en invernadero. Ediciones Mundi Prensa. Colegio de Postgraduados. México DF, México. 265 p.

Bugarin, R; Galvis, A; Sánchez, P; García, D. 2002. Acumulación diaria de materia seca y de potasio en la biomasa aérea total de tomate. Terra 20(4):401-409.

Bertsch, F. 1998. La fertilidad de los suelos y su manejo. Asociación Costarricense de la Ciencia del Suelo. San José, Costa Rica. 157 p.

Bertsch, F. 2003. Absorción de nutrimentos por los cultivos. Asociación Costarricense de la Ciencia del Suelo. San José, Costa Rica. 307 p.
Caravaca, P. 2008. Tomate Costa Rica. Servicio Información de Mercados. Dirección Mercadeo y Agroindustria. Consejo Nacional de la Producción. Boletín $\mathrm{N}^{\circ} 1.10$ p.

Cerdas, MM; Montero, ME. 2002. Manual de manejo poscosecha de tomate. Ministerio de Agricultura y Ganadería. Universidad de Costa Rica. FITTACORI. San José, Costa Rica. 69 p.

Cook, R; Calvin, L. 2005. Greenhouse tomatoes change the dynamics of the North American Fresh Tomato Industry. USDA. Economic Research Report $\mathrm{N}^{\circ} 2$. $81 \mathrm{p}$.

Costa, M; Heuvelink, E. 2006. Today's worlwide tomato production. Fruit \& Veg Tech: International Suppliers Guide 2007. Amsterdam. p. 14-16.

FAO. sf. El cultivo protegido en clima mediterráneo. Estudio Producción y Protección Vegetal 90. FAO. Roma, Italia. 320 p.

Fayad, JA; Fontes, PCR; Cardoso, AA; Finger, FL; Ferreira, FA. 2002. Absorção de nutrientes pelo tomateiro cultivado sob condições de campo e de ambiente protegido. Horticultura Brasileira 20(1):90-94.

Gómez, O; Casanova, A; Laterrot, H; Anais, G. 2000. Mejora genética y manejo del cultivo del tomate para la producción en el Caribe. Instituto de Investigaciones Hortícolas "Liliana Dimitrova". La Habana, Cuba. $159 \mathrm{p}$.

Handreck, K; Black, N. 2002. Growing media for ornamental plants and turf. 3 ed. UNSW Press. Sidney, Australia. $542 \mathrm{p}$.

HAIFA Nutri-Net, 2008. Nutrient requirements - tomatoes (en línea). Consultado 18 ene. 2008. Disponible en http://www.haifa-nutrinet.com/default.asp

Imas, P. 2009. Manejo de nutrientes por fertirriego en sistemas frutihortícolas (en línea). Consultado 16 jul. 2009. Disponible en http://www.itescam.edu.mx/ principal/sylabus/fpdb/recursos/r16614.DOC

Infostat. 2002. InfoStat/Profesional v.1.1. Facultad Ciencias Agropecuarias, Universidad Nacional de Córdoba. Argentina.

Marín, M; Araya, G; Valverde JC. 2008. Respuesta del cultivo de tomate (Lycopersicun esculentum) a la fertilización fraccionada en tres programas de nutrición. In 54 Reunión Anual PCCMCA (Memoria de resúmenes). San José, Costa Rica. p. 94.

Marín, F. 2010. Cuantificación y valoración de estructuras y procesos de producción agrícola bajo ambientes protegidos en Costa Rica. FITTACORI. San José, Costa Rica. 34 p.

AGRONOMÍA MESOAMERICANA 23(1):117-128 2012 
Molina, E. 2006. Principios generales de la fertirrigación en la nutrición de plantas. In Seminario Fertirrigación de Cultivos. Memoria. San José, Costa Rica. p. 5164.

Nicola, S; Tibaldi, G; Fontana, E. 2009. Tomato production systems and their application to the tropics. Proc. IS on tomato in the tropics. Acta Horticulturae 821:2733.

Quesada, G; Bertsch, F. 2009. Determinación de la curva de absorción de nutrimentos para el híbrido de tomate FB-17. Informe parcial Proyecto 736-A6-101. Universidad de Costa Rica. San José, CR. 29 p.

Sánchez, F. 2004. Descripción de paquetes tecnológicos de producción comercial de jitomate en hidroponía bajo invernadero. In Salazar R; Navas G; Rojano A. eds. III Curso Internacional de Invernaderos. Guadalajara, México. PUIMECI. 38 p.
Scaife, A; Bar-Yosef, B. 1995. Nutrient and fertilizer management in field grown vegetables. International Potash Institute. Basilea, Suiza. 104 p.

SEPSA (Secretaría Ejecutiva de Planificación Sectorial Agropecuaria). 2011. Boletín estadístico agropecuario $n^{\circ}$ 21. San José, CR. Secretaría Ejecutiva de Planificación Sectorial Agropecuaria. Área de Estudios Económicos e Información (en línea). 182 p. Consultado 18 set. 2011. Disponible en http://www.infoagro.go.cr

Snyder, RG. 2006. Guía del cultivo del tomate en invernaderos. Mississippi, US. Servicio de Extensión de la Universidad Estatal de Mississippi. USA. Publicación No 2419. 24 p.

StatSoft Inc. 2001. STATISTICA (Data Analysis Software System), v.6.0. www.statsoft.com.

Vallejo, FA. 1999. Mejoramiento genético y producción de tomate en Colombia. Universidad Nacional de Colombia. Cali, Colombia. 216 p. 